Case Report

\title{
A Thought-Provoking Case of Successfully Treated Carcinoma of the Head of the Pancreas with Metachronous Lung Metastasis: Impact of Distal Spleno-Renal Shunt for Regional Invasion on Long-Term Period after Pancreaticoduodenectomy
}

\author{
Ryuhei Aoyama $(\mathbb{D}$, Tomohide Hori $(\mathbb{D}$, Hidekazu Yamamoto $(\mathbb{D}$, Hideki Harada $(\mathbb{D}$, \\ Michihiro Yamamoto $\left(\mathbb{D}\right.$, Masahiro Yamada $\left(\mathbb{D}\right.$, Takefumi Yazawa $\mathbb{D}$, Ben Sasaki $\mathbb{D}^{\text {, }}$ \\ Masaki Tani ${ }^{D}$, Asahi Sato $\left(\mathbb{D}\right.$, Hikotaro Katsura $\left(\mathbb{D}\right.$, Yasuyuki Kamada $\left(\mathbb{D}\right.$, Ryotaro Tani ${ }^{(D)}$, \\ Yudai Sasaki $(\mathbb{D}$, and Masazumi Zaima
}

Department of Surgery, Shiga General Hospital, Moriyama, Japan

Correspondence should be addressed to Tomohide Hori; horitomo55office@yahoo.co.jp

Received 21 October 2020; Accepted 14 May 2021; Published 29 May 2021

Academic Editor: Claudio Feo

Copyright ( 2021 Ryuhei Aoyama et al. This is an open access article distributed under the Creative Commons Attribution License, which permits unrestricted use, distribution, and reproduction in any medium, provided the original work is properly cited.

\begin{abstract}
When performing pancreaticoduodenectomy with resection of the confluence of the superior mesenteric vein and portal vein, division of the splenic vein may cause sinistral portal hypertension resulting in gastrointestinal bleeding, splenic congestion, and hypersplenism. To prevent these adverse events, it is important to intentionally decompress the splenic vein. This report is of a 68 -year-old woman with stage IA carcinoma of the head of the pancreas who survived for more than six years following tumor resection and pancreaticoduodenectomy and distal splenorenal shunt. A 68-year-old woman was diagnosed with carcinoma of the head of the pancreas that involved the confluence of the superior mesenteric vein, portal vein, and splenic vein. No unresectable cancer sites or distant metastases were detected. Pancreaticoduodenectomy with resection of the confluence of the superior mesenteric vein and portal vein was performed. The superior mesenteric vein and portal vein were anastomosed in the end-to-end fashion, and the remnant splenic vein was anastomosed to the superior aspect of the left renal vein in the end-toside fashion. At 22 months after the initial surgery, the patient underwent partial lung resection for a metachronous lung metastasis. For 6 years after the initial surgery, the venous reconstructions have maintained their patency without any obstruction of splenic venous flow, and the patient has remained in good health without further metastases or recurrences. This case has shown the importance of early diagnosis of carcinoma of the head of the pancreas, as appropriate and timely surgical management can result in good outcome. This patient responded well and remains alive six years following pancreaticoduodenectomy and preservation of the spleen with the use of a distal splenorenal shunt.
\end{abstract}

\section{Introduction}

Pancreas cancer is the fourth leading cause of cancer-related death in developed countries [1], and the frequency currently increases with unknow reason [2]. Pancreatic cancer often accompanies with distant metastasis and generally shows poor prognosis [3-6]. Pancreatic cancer remains one of the most lethal malignancies with a 5-year survival rate of 5$10 \%[7,8]$. Pancreatic cancer is often already incurable at the time of diagnosis and have a median overall survival of 6-12 months even with palliative chemotherapy [7]. The risk of postoperative recurrence is high, and a 5-year survival rate after surgery was documented as approximately 30\% [7]. Hence, multidisciplinary treatment should be considered [8].

Pancreatic cancer located in the pancreatic head and/or uncus easily invades the superior mesenteric vein and/or portal vein [9-11]. Although venous reconstruction of the superior mesenteric vein and portal vein is widely accepted, it 
remains controversial whether there is a validity of intentional decompression of the splenic vein in cases requiring diversion of the splenic vein [12-20].

W. Dean Warren (1924-1989) was a pioneer in surgery for portal hypertension [21], and he focused on therapeutic potential of distal splenorenal shunt for portal hypertension and variceal bleeding [22-26]. He first described surgical procedure using distal splenorenal shunt in 1967 [27], and "the Warren shunt," is a vascular surgery of distal splenorenal shunt. Briefly, the splenic vein is detached from the superior mesenteric vein and portal vein and is subsequently reattached to the left renal vein [21].

Division and ligation of the splenic vein during pancreaticoduodenectomy is often associated with the development of sinistral portal hypertension, which results in gastrointestinal bleeding, splenic congestion, and hypersplenism over the long term $[12,13]$. Therefore, optimal management of the splenic vein is required [13]. The individual vascular anatomy dictates whether intentional preservation of the splenic venous drainage via the inferior mesenteric vein or other venous tributaries is sufficient to prevent sinistral portal hypertension $[13,14]$; some surgeons suggest that splenic venous reconstruction is important for long-term splenic decompression [15-20].

This report describes a 68-year-old woman with stage IA carcinoma of the head of the pancreas who survived for more than six years following tumor resection and pancreaticoduodenectomy with the use of a distal splenorenal shunt to preserve the spleen.

\section{Case Presentation}

A 68-year-old woman was referred to our hospital for the investigation of elevated serum levels of amylase and lipase. Enhanced computed tomography (CT) detected a hypovascular mass (18 mm diameter) in the pancreatic head (Figures 1(a) and 1(b)). The main pancreatic duct of the distal pancreatic parenchyma was substantially dilated (Figures 1(a) and 1(b)). Endoscopic retrograde and magnetic resonance cholangiopancreatographies showed severe stenosis of the main pancreatic duct due to the pancreatic tumor, but biliary obstruction was not observed. The diagnosis of carcinoma of the head of the pancreas was made based on the histopathologic examination of an endoscopic ultrasound-guided fine-needle aspiration biopsy. Dynamic CT clearly demonstrated that the carcinoma of the head of the pancreas had invaded the superior mesenteric vein, portal venous trunk, and splenic vein (Figure 1(c)). The inferior mesenteric vein flowed into the superior mesenteric vein rather than the splenic vein (Figure $1(\mathrm{~d})$ ), suggesting that the inferior mesenteric vein was not a viable splenic venous drainage route. There were no findings indicating cancer at an unresectable site or distant metastases. Neoadjuvant chemotherapy was not employed, and a subtotal stomachpreserving pancreaticoduodenectomy with lymphadenectomy was performed. Histopathological assessments revealed that a moderately differentiated invasive ductal carcinoma had invaded the venous wall of the confluence of the superior mesenteric vein, portal venous trunk, and splenic vein. Pan- creatic cancer measuring 1 to $2 \mathrm{~cm}$ is categorized as T1c, and the cancer categorized as T1cN0M0 stage IA in accordance with the tumor-node-metastasis classification [28], although pancreatic cancer growing outside the pancreas and into nearby major blood vessels is categorized as T4 and stage III according to staging system of American Cancer Society [29].

As imaging examinations revealed that the tumor involved the confluence of the superior mesenteric vein, portal venous trunk, and splenic vein (Figures 1(c) and 1(d)), en bloc resection of the confluence of these veins was performed, with simultaneous venous reconstructions (Figure 2). The lower margin of the distal pancreas was incised, and the pancreas was rotated cranially to expose the posterior surface of the splenic vein. The splenic vein was then ligated at the confluence of the superior mesenteric vein and portal vein and dissected toward the pancreatic tail to obtain a sufficient length for anastomosis. The splenic vein was divided as near as possible to the junction of the superior mesenteric vein and portal vein and temporarily clamped (Figure 3(a)). The left renal vein was then prepared for anastomosis on the left side of the superior mesenteric artery and partially clamped (Figure 3(a)). An anastomosis orifice was created on the superior aspect of the left renal vein rather than the ventral aspect (Figure 3(b)). After widening the cut end of the splenic vein by making a small slit in its heel side, the splenic vein and left renal vein were anastomosed in the end-to-side fashion by bilateral fixation sutures (6-0 Prolene; Ethicon, Inc., Cincinnati, USA) (Figure 3(b)). Subsequently, the remnant splenic vein was anastomosed to the superior aspect of the left renal vein in the end-to-side fashion by unabsorbable polypropylene (6-0 Prolene) (Figures 3(c) and $3(\mathrm{~d}))$. After the specimen was removed, the superior mesenteric vein and portal venous trunk were anastomosed in the usual end-to-end fashion by running sutures using unabsorbable polypropylene (5-0 Prolene). The operative time was 348 minutes. The intraoperative blood loss volume was $1,294 \mathrm{~mL}$, and no blood transfusion was required. Although there was temporary ulceration of the gastrojejunostomy, categorized as grade II in accordance with the Clavien-Dindo classification [30], the postoperative course was uneventful.

Histopathological assessments of resected specimen revealed that moderately differentiated invasive ductal carcinoma clearly invaded into venous wall of the confluence of the superior mesenteric vein, portal venous trunk, and splenic vein. Adjuvant chemotherapy with S-1 was administered for 6 months postoperatively. At 22 months after the initial surgery, ground-glass opacity ( $8 \mathrm{~mm}$ in size) was graphically detected in the right lung. The patient underwent partial lung resection for a right lung tumor that was pathologically diagnosed as a lung metastasis originated from the pancreatic cancer (i.e., metachronous metastasis), although graphical and surgical curability were obtained. Chemotherapy with S-1 was resumed for 1 year after the lung resection. The patient has been in good health, with no other oncologic metastases detected during the 6 years since the initial surgery. 


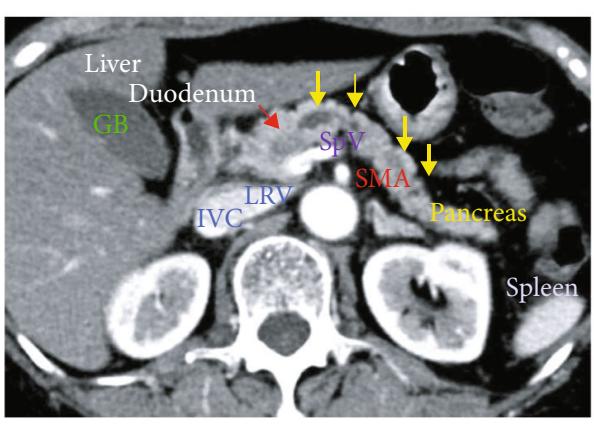

(a)

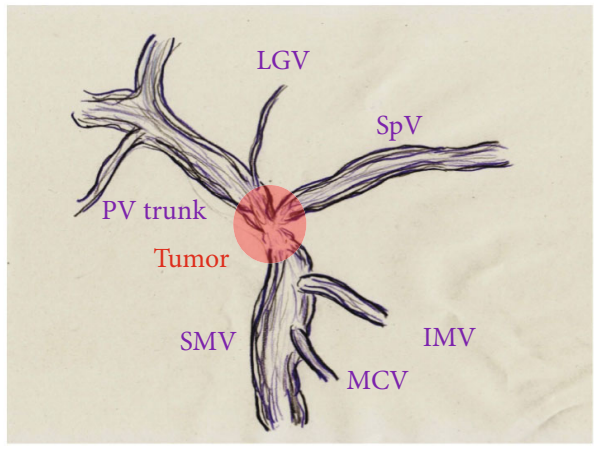

(c)

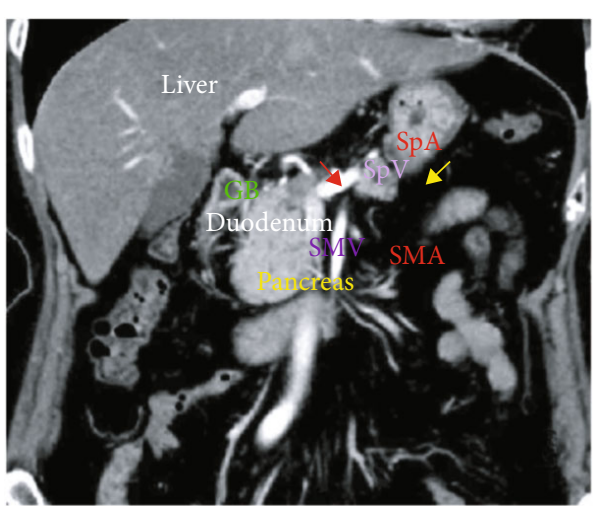

(b)

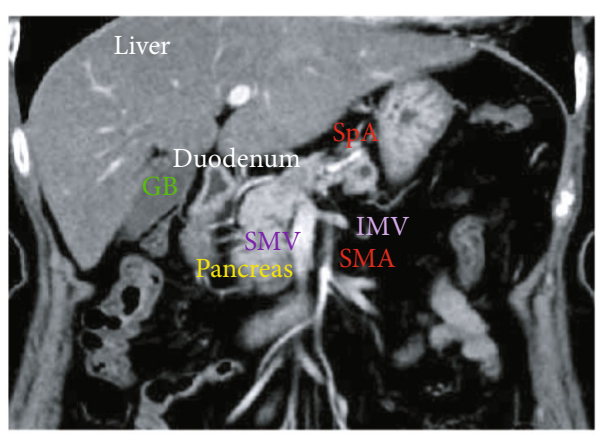

(d)

FIGURE 1: Pancreatic cancer with regional invasion into vessels. (a, b) Enhanced computed tomography shows a hypovascular mass in the pancreatic head (red arrow) and marked dilation of the main pancreatic duct of the distal pancreatic parenchyma (yellow arrows). (c) The pancreatic tumor (red area) markedly involves the confluence of the superior mesenteric vein, portal venous trunk, and splenic vein. (d) The inferior mesenteric vein flows into the superior mesenteric vein, not the splenic vein. There is no evidence of cancer at unresectable sites or distant metastases. GB: gallbladder; IMV: inferior mesenteric vein; LGV: left gastric vein; LRV: left renal vein; IVC: inferior vena cava; MCV: middle colic vein; SMA: superior mesenteric artery; SMV: superior mesenteric vein; SpA: splenic artery; SpV: splenic vein.

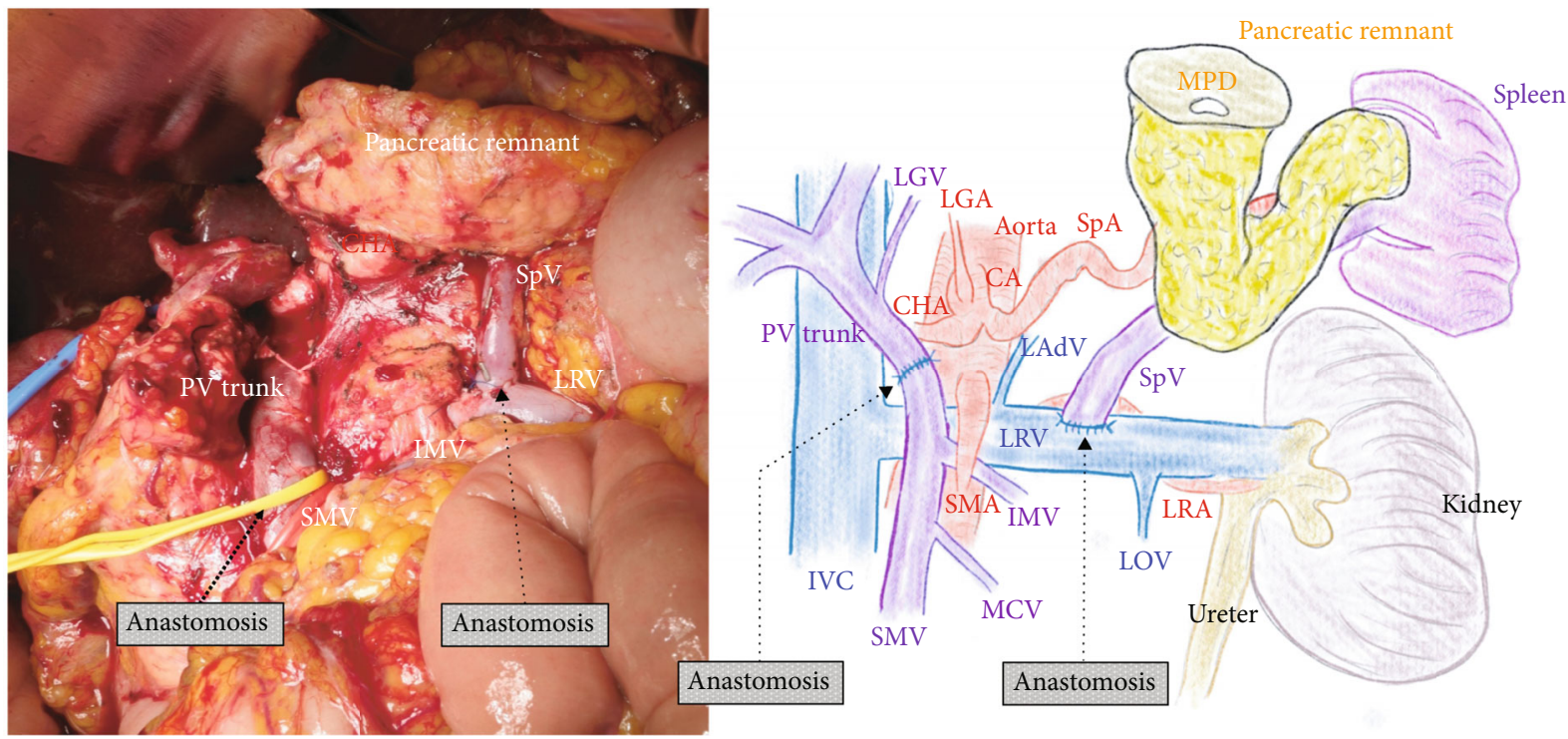

Figure 2: The distal splenorenal shunt. The confluence of the superior mesenteric vein, portal vein, and splenic vein are resected en bloc. The superior mesenteric vein and portal venous trunk are anastomosed in the end-to-end fashion. The remnant splenic vein is anastomosed to the superior aspect of the left renal vein in the end-to-side fashion. CA: celiac artery; CHA: common hepatic artery; IMV: inferior mesenteric vein; LAdV: left adrenal vein; LGA: left gastric artery; LGV: left gastric vein; LOV: left ovarian vein; LRA: left renal artery; LRV: left renal vein; IVC: inferior vena cava; MCV: middle colic vein; MPD: main pancreatic duct; PV: portal vein; SMA: superior mesenteric artery; SMV: superior mesenteric vein; $\mathrm{SpA}$ : splenic artery; $\mathrm{SpV}$ : splenic vein. 


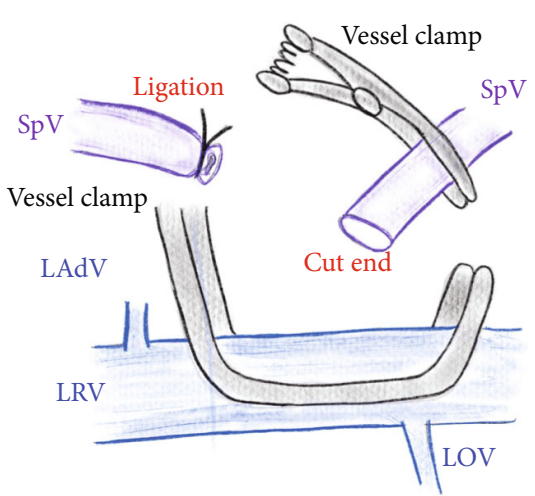

(a)

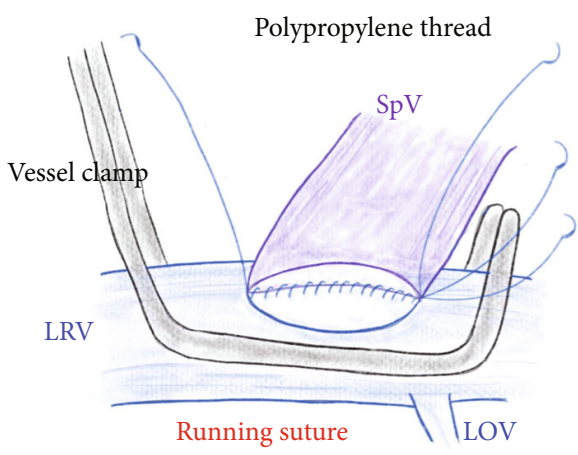

(c)

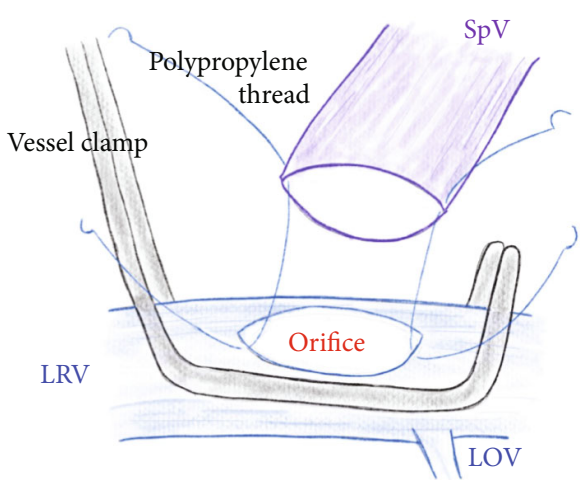

(b)

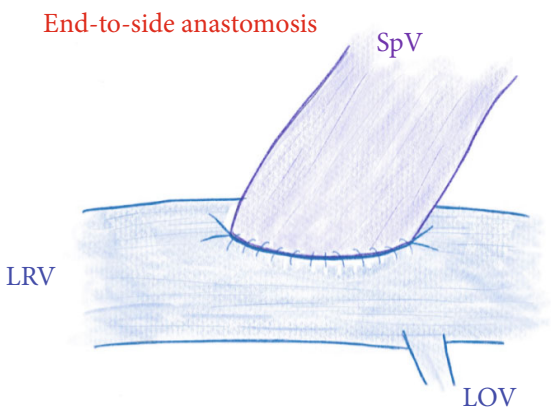

(d)

Figure 3: Surgical procedures. (a) The splenic vein is ligated and cut, and the splenic vein is temporarily clamped. The left renal vein is partially clamped. (b) A venous orifice is made on the superior aspect of the LRV. The splenic vein and left renal vein are set in the endto-side fashion by bilateral fixation sutures. $(c, d)$ The splenic vein is anastomosed to the left renal vein in the end-to-side fashion by unabsorbable polypropylene. LAdV: left adrenal vein; LOV: left ovarian vein; LRV: left renal vein; SpV: splenic vein.

Dynamic CT and upper esophagogastric endoscopy performed 5 years and 6 months after the initial surgery revealed that all venous reconstructions had maintained good patency (Figure 4), and there were no esophageal varices or congestive gastropathy. The platelet counts before surgery and at 5 years and 6 months after the initial surgery were $221,000 / \mu \mathrm{L}$ and $235,000 / \mu \mathrm{L}$, respectively. The estimated splenic volumes before surgery and at 5 years and 6 months after the initial surgery were $38 \mathrm{~mL}$ and $35 \mathrm{~mL}$, respectively.

\section{Discussion}

Intentional decompression of the splenic vein after division of this vein is still controversial [12-20], and a creation of distal splenorenal shunt requires vascular surgical skill. Moreover, postoperative long-term effect of distal splenorenal shunt is unknown, because pancreatic cancer generally accompanies with distant metastasis and shows very poor prognosis [3-6]. Our thought-provoking case suggested intentional decompression of the splenic vein should be considered for the long-term postoperative course, and distal splenorenal shunt during pancreaticoduodenectomy is a powerful tool for carcinoma of the head of the pancreas with regional invasion.

Although venous reconstruction of the superior mesenteric vein and portal vein during pancreaticoduodenectomy for pancreatic cancer is widely accepted, intentional decompression of the splenic vein in case when division of the splenic vein is needed is still controversial [12-20]. It has been clearly demonstrated that division of the splenic vein during pancreaticoduodenectomy causes sinistral portal hypertension, which results in intractable symptoms (e.g., gastrointestinal bleeding, splenic congestion, and hypersplenism) in the long term $[12,13]$. Hence, many surgeons focused on sinistral portal hypertension after pancreaticoduodenectomy with resection of the confluence of superior mesenteric and portal veins, because only a little was previously known [18]. It is currently clarified that pancreaticoduodenectomy with splenic venous division causes variceal formation, bleeding, and thrombocytopenia [18], and therefore, the splenic venous flow is intentionally reconstructed if pancreaticoduodenectomy accompanies with the splenic venous diversion [15-20].

As pancreatic cancer generally has a very poor prognosis [3-6], many surgeons have not payed much attention to the consequence of sinistral portal hypertension on the long- 

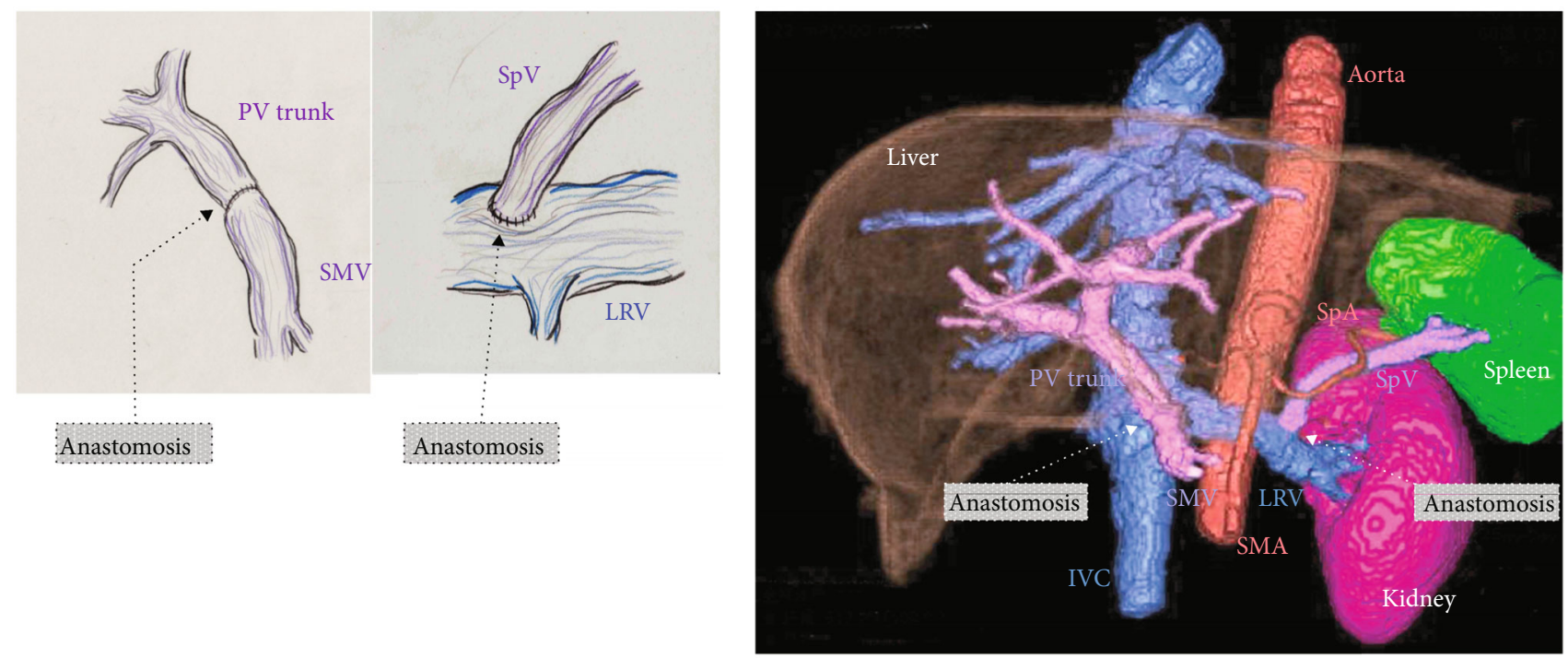

(a)

(b)

FIGURE 4: Long-term patency of the distal splenorenal shunt. (a) Schemas for venous reconstructions during pancreaticoduodenectomy. (b) Three-dimensional imagery at 5 years and 6 months after the initial surgery shows that the distal splenorenal shunt has maintained good patency. The estimated splenic volume is $35 \mathrm{~mL}$. LRV: left renal vein; IVC: inferior vena cava; PV: portal vein; SMA: superior mesenteric artery; SMV: superior mesenteric vein; SpA: splenic artery; SpV: splenic vein.

term postoperative course. However, multimodality therapy has improved the prognosis of pancreatic cancer [31-33], and intentional decompression of the splenic vein is currently considered important for the long-term postoperative course.

A native vein may be the optimal candidate as a drainage route of the splenic vein. The inferior mesenteric vein is useful as a drainage route from the splenic vein $[13,34,35]$, but the inferior mesenteric vein only works as a drainage route when it is connected to the splenic vein. In contrast to other opinions, others suggest that intentional preservation of the inferior mesenteric vein might not prevent splenic congestion [14], as the left gastric vein, middle colic vein, and superior right colic vein arcade may provide adequate drainage from the splenic vein and prevent sinistral portal hypertension [17]. However, the beneficial effect of drainage via these critical veins is greatly affected by individual anatomy [17], and it is often difficult to preserve these critical veins in patients with advanced pancreatic cancer.

Most surgeons currently believe that the splenic venous flow must be reconstructed in cases where the splenic vein is divided [15-20]. Although some venous reconstructions (e.g., anastomoses to the inferior mesenteric vein or direct implantation to the superior mesenteric vein and portal vein) have been already documented $[12,15,20]$, these reconstructions have limitations regarding feasibility and long-term patency. Hence, we consider that the splenic vein should be antegradely reconstructed into a thick vein (i.e., the left renal vein), and so we created a distal splenorenal shunt for splenic venous reconstruction since 2014.

Warren was a pioneer in surgery for portal hypertension and variceal bleeding and originally published the distal splenorenal shunt in 1967 as an operative procedure for bleeding esophageal varices [27]. Warren originally established the distal splenorenal shunt for patients with cirrhosis, and operation in cirrhotic patient needs advanced surgical skill in handling the splenic vein with high pressure and splenopancreatic disconnection in necessary to maintain selectivity of portal perfusion. Creation of distal splenorenal shunt in noncirrhotic patients is much easier and safer, because these troublesome procedures are unnecessary. We therefore considered the distal splenorenal shunt safe and feasible in patients without liver cirrhosis. Other surgeons employed distal splenorenal shunt for intentional decompression of the splenic vein during pancreatic surgery in cirrhotic patient [36], we have a clear impression that a creation of splenorenal shunt is easier than that in cirrhotic patient. Splenorenal shunt has a safety and feasibility for intentional decompression of the splenic vein. On the other hand, we made distal splenorenal shunt first, in order to avoid even a subtle splenic congestion during surgery. We did not use vessel prosthesis, because of pancreatic juice-related complication after surgery.

To our knowledge, there are only few reported cases of splenic venous reconstruction via a distal splenorenal shunt [12, 37-39]. Moreover, no report has focused on the longterm effects of a distal splenorenal shunt to prevent sinistral portal hypertension, as pancreatic cancer has a very poor prognosis [3-6]. In the present case, we used a distal splenorenal shunt for splenic venous reconstruction during radical surgery for carcinoma of the head of the pancreas with regional invasion, and close long-term follow-up revealed venous anastomotic patency with no recurrence. Currently, tumor biology and risk factor for pancreatic cancer are detected $[40,41]$. An exceptional long-term survival of our patient might be probably related to a favorable tumor biology.

In conclusion, this case has shown the importance of early diagnosis of carcinoma of the head of the pancreas, 
as appropriate and timely surgical management can result in good outcome. This patient responded well and remains alive six years following pancreaticoduodenectomy and preservation of the spleen with the use of a distal splenorenal shunt.

\section{Data Availability}

All data were clearly shown in figures and text.

\section{Ethical Approval}

Data in this case report were retrospectively evaluated. This report was approved by the Institutional Review Board of our institution.

\section{Consent}

The patient involved in this study provided written informed consent authorizing the use and disclosure of her protected health information.

\section{Conflicts of Interest}

None of the authors have any financial conflicts of interest to declare.

\section{Authors' Contributions}

R. Aoyama and T. Hori collected the data and analyzed the data. T. Hori wrote the manuscript. R. Aoyama and T. Hori contributed equally to this work. All authors discussed therapeutic options, reviewed previous papers, and provided important opinions. M. Zaima and T. Hori supervised this report.

\section{References}

[1] S. Williams, "Tapping into nerve conversations," Science, vol. 248, no. 4955, p. 555, 1990.

[2] M. Ducreux, T. Seufferlein, J. L. Van Laethem et al., "Systemic treatment of pancreatic cancer revisited," Seminars in Oncology, vol. 46, no. 1, pp. 28-38, 2019.

[3] V. H. Coupland, H. M. Kocher, D. P. Berry et al., "Incidence and survival for hepatic, pancreatic and biliary cancers in England between 1998 and 2007," Cancer Epidemiology, vol. 36, no. 4, pp. e207-e214, 2012.

[4] R. Siegel, D. Naishadham, and A. Jemal, "Cancer statistics, 2013,” CA: a Cancer Journal for Clinicians, vol. 63, no. 1, pp. 11-30, 2013.

[5] A. Carrato, A. Falcone, M. Ducreux et al., "A systematic review of the burden of pancreatic cancer in Europe: real-world impact on survival, quality of life and costs," Journal of Gastrointestinal Cancer, vol. 46, no. 3, pp. 201-211, 2015.

[6] M. Ducreux, A. S. Cuhna, C. Caramella et al., "Cancer of the pancreas: ESMO clinical practice guidelines for diagnosis, treatment and follow-up," Annals of Oncology, vol. 26, pp. 56-68, 2015.

[7] M. J. Monroy-Iglesias, S. Dolly, D. Sarker, K. Thillai, M. Van Hemelrijck, and A. Santaolalla, "Pancreatic cancer exposome profile to aid early detection and inform prevention strategies," Journal of Clinical Medicine, vol. 10, no. 8, p. 1665, 2021.

[8] J. D. Mizrahi, R. Surana, J. W. Valle, and R. T. Shroff, "Pancreatic cancer," Lancet, vol. 395, no. 10242, pp. 2008-2020, 2020.

[9] D. O'Reilly, L. Fou, E. Hasler et al., "Diagnosis and management of pancreatic cancer in adults: a summary of guidelines from the UK National Institute for Health and Care Excellence," Pancreatology, vol. 18, no. 8, pp. 962-970, 2018.

[10] P. Charalambous, D. Moris, G. S. Karachaliou et al., "The efficacy and safety of the open approach irreversible electroporation in the treatment of pancreatic cancer: a systematic review," European Journal of Surgical Oncology, vol. 46, no. 9, pp. 1565-1572, 2020.

[11] H. Amano, F. Miura, N. Toyota et al., "Is pancreatectomy with arterial reconstruction a safe and useful procedure for locally advanced pancreatic cancer?," Journal of Hepato-BiliaryPancreatic Surgery, vol. 16, no. 6, pp. 850-857, 2009.

[12] P. Addeo, G. Nappo, E. Felli, C. Oncioiu, F. Faitot, and P. Bachellier, "Management of the splenic vein during a pancreaticoduodenectomy with venous resection for malignancy," Updates in Surgery, vol. 68, no. 3, pp. 241-246, 2016.

[13] F. Bianco, C. Sassaroli, P. Delrio, S. De Franciscis, and G. Romano, "Vascular resection in pancreaticoduodenectomy: is it worthwhile?," Current Drug Targets, vol. 13, no. 6, pp. 772-780, 2012.

[14] M. Hattori, T. Fujii, S. Yamada et al., "Significance of the splenic vein and its branches in pancreatoduodenectomy with resection of the portal vein system," Digestive Surgery, vol. 32, no. 5, pp. 382-388, 2015.

[15] N. Ferreira, E. Oussoultzoglou, P. Fuchshuber et al., "Splenic vein-inferior mesenteric vein anastomosis to lessen left-sided portal hypertension after pancreaticoduodenectomy with concomitant vascular resection," Archives of Surgery, vol. 146, no. 12, pp. 1375-1381, 2011.

[16] Y. Ono, K. Matsueda, R. Koga et al., "Sinistral portal hypertension after pancreaticoduodenectomy with splenic vein ligation," The British Journal of Surgery, vol. 102, no. 3, pp. 219228, 2015.

[17] M. Tanaka, H. Ito, Y. Ono et al., "Impact of portal vein resection with splenic vein reconstruction after pancreatoduodenectomy on sinistral portal hypertension: who needs reconstruction?," Surgery, vol. 165, no. 2, pp. 291-297, 2019.

[18] S. Mizuno, H. Kato, H. Yamaue et al., "Left-sided portal hypertension after pancreaticoduodenectomy with resection of the portal vein/superior mesenteric vein confluence in patients with pancreatic cancer: a project study by the Japanese Society of Hepato-Biliary-Pancreatic Surgery," Annals of Surgery, vol. Publish Ahead of Print, 2019in press.

[19] M. H. G. Katz, J. E. Lee, P. W. T. Pisters, R. Skoracki, E. Tamm, and J. B. Fleming, "Retroperitoneal dissection in patients with borderline resectable pancreatic cancer: operative principles and techniques," Journal of the American College of Surgeons, vol. 215, pp. 11-18, 2012.

[20] K. Misuta, H. Shimada, Y. Miura et al., "The role of splenomesenteric vein anastomosis after division of the splenic vein in pancreatoduodenectomy," Journal of Gastrointestinal Surgery, vol. 9, no. 2, pp. 245-253, 2005.

[21] H. C. Polk Jr., D. Levi, and D. G. Hutson, "W Dean Warren, MD: iron hand and principles of steel," Journal of the American College of Surgeons, vol. 228, no. 4, pp. 708-714, 2019. 
[22] W. D. Warren, J. M. Henderson, W. J. Millikan et al., "Distal splenorenal shunt versus endoscopic sclerotherapy for longterm management of variceal bleeding. Preliminary report of a prospective, randomized trial," Annals of Surgery, vol. 203, no. 5 , pp. $454-462,1986$.

[23] W. J. Millikan Jr., W. D. Warren, J. M. Henderson et al., "The Emory prospective randomized trial: selective versus nonselective shunt to control variceal bleeding. Ten year follow-up," Annals of Surgery, vol. 201, no. 6, pp. 712-722, 1985.

[24] B. M. Nordlinger, D. F. Nordlinger, J. T. Fulenwider et al., "Angiography in portal hypertension: clinical significance in surgery," American Journal of Surgery, vol. 139, no. 1, pp. 132-141, 1980.

[25] W. D. Warren, W. J. Millikan Jr., J. M. Henderson, M. E. Rasheed, and A. A. Salam, "Selective variceal decompression after splenectomy or splenic vein thrombosis. With a note on splenopancreatic disconnection," Annals of Surgery, vol. 199, no. 6, pp. 694-702, 1984.

[26] W. D. Warren, W. J. Millikan Jr., J. M. Henderson et al., “Ten years portal hypertensive surgery at Emory. Results and new perspectives," Annals of surgery, vol. 195, no. 5, pp. 530-542, 1982.

[27] W. D. Warren, R. Zeppa, and J. J. Fomon, "Selective transsplenic decompression of gastroesophageal varices by distal splenorenal shunt," Annals of Surgery, vol. 166, no. 3, pp. 437-455, 1967.

[28] Union for International Cancer Control (UICC) (US), TNM classification of malignant tumors, Wiley Blackwell, New York, 8th edition, 2017.

[29] American Joint Committee on Cancer (US), "Exocrine Pancreas," in AJCC Cancer Staging Manual, p. 337, Springer, New York, 8th edition, 2017.

[30] P. A. Clavien, J. Barkun, M. L. de Oliveira et al., "The ClavienDindo classification of surgical complications: five-year experience," Annals of Surgery, vol. 250, no. 2, pp. 187-196, 2009.

[31] A. G. Raufi, G. A. Manji, J. A. Chabot, and S. E. Bates, "Neoadjuvant treatment for pancreatic cancer," Seminars in Oncology, vol. 46, no. 1, pp. 19-27, 2019.

[32] J. P. Neoptolemos, J. Kleeff, K. Michl, E. Costello, W. Greenhalf, and D. H. Palmer, "Therapeutic developments in pancreatic cancer: current and future perspectives," Nature Reviews. Gastroenterology \& Hepatology, vol. 15, no. 6, pp. 333-348, 2018.

[33] I. Garrido-Laguna and M. Hidalgo, "Pancreatic cancer: from state-of-the-art treatments to promising novel therapies," Nature Reviews. Clinical Oncology, vol. 12, no. 6, pp. 319334, 2015.

[34] C. H. Pilgrim, S. Tsai, P. Tolat et al., "Optimal management of the splenic vein at the time of venous resection for pancreatic cancer: importance of the inferior mesenteric vein," Journal of Gastrointestinal Surgery, vol. 18, no. 5, pp. 917-921, 2014.

[35] D. Arnaoutakis and F. Eckhauser, "Safety and effectiveness of splenic vein to inferior mesenteric vein anastomosis during pancreaticoduodenectomy: comment on 'splenic veininferior mesenteric vein anastomosis to lessen left-sided portal hypertension after pancreaticoduodenectomy with concomitant vascular resection'," Archives of Surgery, vol. 146, no. 12, pp. 1381-1382, 2011.

[36] G. M. Ettorre, G. B. Levi Sandri, M. Colasanti, E. de Werra, and P. Lepiane, "Distal pancreatectomy with splenorenal shunt to preserve spleen in a cirrhotic patient," Annals of hepatobiliary-pancreatic surgery, vol. 21, no. 2, pp. 93-95, 2017.

[37] K. K. Christians, K. Riggle, R. Keim et al., "Distal splenorenal and temporary mesocaval shunting at the time of pancreatectomy for cancer: initial experience from the Medical College of Wisconsin," Surgery, vol. 154, no. 1, pp. 123-131, 2013.

[38] M. I. Chavez, S. Tsai, C. N. Clarke et al., "Distal splenorenal and mesocaval shunting at the time of pancreatectomy," Surgery, vol. 165, no. 2, pp. 298-306, 2019.

[39] F. Oehme, M. Distler, B. Müssle, C. Kahlert, J. Weitz, and T. Welsch, "Results of portosystemic shunts during extended pancreatic resections," Langenbeck's Archives of Surgery, vol. 404, no. 8, pp. 959-966, 2019.

[40] A. McGuigan, P. Kelly, R. C. Turkington, C. Jones, H. G. Coleman, and R. S. McCain, "Pancreatic cancer: a review of clinical diagnosis, epidemiology, treatment and outcomes," World Journal of Gastroenterology, vol. 24, no. 43, pp. 4846-4861, 2018.

[41] M. Felsenstein and R. H. Hruban, "New developments in the molecular mechanisms of pancreatic tumorigenesis," Advances in anatomic pathology, vol. 25, no. 2, pp. 131-142, 2018. 